\title{
Corrientes educativas que han permeado la formación de enfermería en la Escuela Nacional de Enfermería y Obstetricia
}

\author{
Araceli Sánchez Ramos*
}

RESUMEN

Este artículo presenta una revisión de los modelos pedagógicos que tradicionalmente se han usado en la formación del profesional de enfermería de la Escuela Nacional de Enfermería y Obstetricia de la Universidad Nacional Autónoma de México (UNAM) y también presenta cómo esos modelos han influido en las características del ejercicio de la profesión. El modelo enseñanza-aprendizaje se ha venido presentando en diversas modalidades, como la tradicionalista, la conductista y actualmente, se pretende que sea constructivista. Finalmente se puede concluir en el presente escrito que a través de la historia de la enfermería se puede observar que el patrón curricular está orientado al desarrollo de habilidades manuales y procedimentales en los que los alumnos, desde el nivel preescolar hasta el superior, conservan un enfoque educativo tradicional con el que se ha formado la mayor parte del personal de la salud. Consecuentemente este modelo educativo genera la pasividad del alumno resaltando la autoridad y el poder del profesor como poseedor del conocimiento.

Palabras clave: Corrientes educativas, formación de enfermería.

\section{Educational flows that have permeated the nursing education in the National School of Nursing and Midwifery}

\begin{abstract}
This article presents a review of pedagogical models that traditionally have been used in professional formation of nurses from the National School of Nursing and Midwifery (ENEO) of the National Autonomous University of Mexico (UNAM) and how these models have influenced the characteristics of the practice of the profession the teaching learning model has been presented in different modalities, such as traditionalist, behavioral and currently intended to be constructive. Finally it can be concluded in this paper is that through the history of nursing can be seen that the curricular pattern is oriented to the development of manuals and procedural skills, where students from preschool to upper retain a traditional educational approach which has trained the majority of the health care personnel. Consequently this educational model generates student passivity highlighting the power and authority of the teacher as the possessor of knowledge.
\end{abstract}

Key words: Educational flows, nursing education.

* Instituto Nacional de Neurología “Manuel Velasco Suárez”.

Recibido para publicación: 11/03/2014. Aceptado: 06/05/2014.

Correspondencia: MEE. Araceli Sánchez Ramos.

E-mail: sanchez-araceli1@hotmail.com

Este artículo puede ser consultado en versión completa en http://www.medigraphic.com/enfermerianeurologica 
$\mathrm{D}$ esde sus inicios, la enfermería en México ha enfrentado grandes desafíos ante los cambios sociales, económicos demográficos y epidemiológicos, así como de desigualdad de género, en educación, nuevas formas de consumo y comunicación; además de cambios ambientales, de urbanización, avances de la tecnología y entrada en vigor del tratado de libre tránsito de profesionales. Asimismo, la pedagogía de la enfermería, desde el inicio de la formación de este recurso, responde a las condicionantes propias de los factores que han determinado el desarrollo de la profesión, tales como la condición femenina, la condición de las comunidades de religiosas, las características de la vida militar y la perspectiva médica. Por ello, es importante hacer un recorrido en el pasado del ámbito educativo de la enfermería para conocer la evolución pedagógica y las corrientes educativas que han permeado la disciplina y, conocer así, cómo se van adecuando a los cambios socioculturales de México.

La educación en el ámbito de enfermería se inició con las escuelas de parteras, debido a que en esas épocas, las mujeres morían principalmente por complicaciones en el embarazo, parto y puerperio; esta problemática de salud trajo consigo la necesidad de atención sustentada en los aspectos médicos-tecnológicos que prácticamente estaban centrados en la adquisición de habilidades técnicas y manuales para ser ejecutada en diversos procedimientos de forma automatizada y rutinaria. ${ }^{1,2}$

La enfermería se había considerado hasta entonces un arte, pero Florence Nightingale rechazó estas ideas; luchó por integrar esta disciplina en un sistema interdependiente, lo que le permitió elaborar con las limitaciones de la ciencia de esa época, una doctrina que durante más de un siglo fue técnica, dependiente de la medicina y también de la enfermedad. Aunque todavía no hablamos de una enfermería como la actual, se puede decir que en este momento de la historia se establecieron las bases para el desarrollo de una enfermería profesional y científica.

Mientras este nuevo concepto de la enfermería se iba extendiendo en muchos países de Europa, en México estábamos apenas saliendo de la guerra de independencia y como consecuencia, estábamos reorganizando nuestro país. Asimismo durante la época colonial, los cuidados enfermeros estuvieron a cargo de las órdenes religiosas; esto continuó así hasta que el 2 de febrero de 1861, Benito Juárez publicó un decreto en el que se transformaron los hospitales y centros de beneficencia. Posteriormente, el 26 de febrero de 1863 decretó la extinción de las comunidades religiosas en toda la república que temporalmente habían quedado a cargo de los enfermos de nuestro país, pero en 1874 Lerdo de Tejada las expulsó definitivamente de México dando origen a nuestro propio "período obscuro de la enfermería".
Para principios del siglo XX el Dr. Liceaga, designado por el presidente de la República, comenzó el ambicioso proyecto del Hospital General y la primera Escuela de Enfermería del mismo, que fueron inaugurados en 1905 y 1907 respectivamente; con esto se inició así una evolución de la profesión que nuevamente fue interrumpida por la revolución. ${ }^{3}$

Una vez reorganizado el país después - en la primera mitad del siglo XX- hubo aproximadamente 30 años de un desarrollo creciente en la enfermería; el Estado asumió la responsabilidad de la salud pública y se crearon organismos como el IMSS, ISSSTE, etcétera. Asimismo se construyeron gran cantidad de hospitales, por lo que en las siguientes décadas, la enfermería tomó conciencia de la necesidad de una preparación a nivel licenciatura, que elevara el conocimiento científico de la enfermera y permitiera reforzar el trabajo de esos hospitales. Fue la ENEO de la UNAM, fundada en 1945, quien incorporó en 1968, la licenciatura en enfermería. Hoy en día, la licenciatura en enfermería se imparte en diferentes instituciones estatales en México, así como en algunas universidades privadas. ${ }^{4}$

A través de la historia de la enfermería se puede observar que la educación estaba orientada en la enseñanza tradicional; en ella, los aspectos cognitivos fueron supervalorados y los efectivos y culturales subestimados, es decir, el aprendizaje se reducía a repetir y memorizar, donde la acción del alumno estaba limitada a la palabra que se fija y repite, conformando una personalidad pasiva y dependiente. Las alumnas de enfermería tenían poco margen para pensar y elaborar conocimientos porque sólo se exigía memorización y, por tanto, las tendencias pedagógicas tradicionales contribuían a la preservación de esta práctica asistencial, renuente al análisis crítico; ello separa a la teoría de la práctica, de una forma rígida e inflexible que frena la ejecución de acciones efectivas de cambio. De hecho, la educación muchas veces está disociada de la realidad y la utilización de sus resultados ha sido extremadamente valorizada cuantitativa y no cualitativamente. ${ }^{5}$

Está claro que las características de aprendizaje adquiridas durante nuestra formación las replicamos; esto no quiere decir que sea lo mejor. Sin embargo, continuamos con ellas, ya que los procedimientos y técnicas de enfermería llevan una secuencia lógica que va desde lo simple a lo complicado; la memorización y la verbalización son los elementos esenciales para permitir la ejecución de la intervención y así, nuevamente, caemos en la observación y la medición de lo aprendido; el margen de error está presente cuando se omite o altera el orden de los pasos. ${ }^{6,7}$

Asimismo al revisar el plan de estudios del desarrollo educativo de la licenciatura en enfermería en la escuela Nacional de Enfermería y Obstetricia (ENEO) de la Universidad Nacional Autónoma de México (UNAM) se puede 
observar que su reto ha sido construirse como disciplina científica y brindarle a la sociedad un producto profesional de alta calidad, cuyo objetivo sea el cuidado de la salud de todas las personas, sin embargo, el andar educativo de la profesión en esta escuela ha estado regido por el enfoque tradicionalista y conductista, lo que reduce la capacidad de análisis y crítica en los estudiantes y profesionales. Hay que reconocer que el conductismo ha sido aplicado a la formación de enfermeras, tal vez porque alcanzó el auge en los setenta, cuando también se había comenzado la licenciatura en la carrera de enfermería.

De este modo el modelo conductista es, en esencia, el mismo modelo tradicional de enseñanza pero enmarcado ahora, en unos principios científicos dados por las investigaciones de Pavlov y Skinner, principalmente. En este modelo se busca que el educando "haga" acríticamente las actividades programadas. La comunicación es la misma que en el modelo tradicional con un nuevo elemento: la retroalimentación o respuesta del receptor en el que el estudiante participa sólo como instrumento de comprobación de la existencia de la respuesta esperada.

Es importante señalar que la ENEO de la UNAM ha sido pionera de muchos cambios educativos siempre en aras de un mejoramiento en la calidad de enseñanza y, por ende, en el tipo de profesional que forma; además, busca proporcionarle al estudiante y a los futuros profesionales las herramientas necesarias para desenvolverse en el mercado laboral y que aquél sea competitivo, enfatizando el desarrollo de un estudiante analítico, crítico y constructor de sus propios procesos educativos en donde el papel del docente sea más bien de acompañante experto de esos procesos. ${ }^{8}$

En el modelo educativo del plan de estudios de licenciatura en enfermería y obstetricia de la ENEO, UNAM, que fue actualizado en año 2000, aunque explícitamente no lo menciona, se infiere que está conformado por un enfoque constructivista del aprendizaje, el cual se destaca por ser un enfoque psicológico cuya premisa es considerar al proceso de enseñanza y aprendizaje como un generador de saberes que parten de las experiencias y los conocimientos previos de cada individuo. Las estrategias para la enseñanza de la enfermería se elaboran partiendo de la anterior premisa, pero sabiendo que al enseñar bajo la metodología educativa constructivista, el educando ocupa una posición protagónica en su propio aprendizaje y el docente es un facilitador del proceso. En otras palabras, en el proceso formativo el estudiante expresa ideas, resuelve dudas, va adquiriendo experiencias y construyendo su propio conocimiento. ${ }^{9}$

Para los alumnos, sin embargo, representa un desafío, porque les es difícil desarrollar habilidades de estudio independientes; esta situación es comprensible, ya que en nuestra sociedad mexicana las instituciones educativas desde el nivel preescolar hasta el superior conservan un enfoque educativo tradicional con el cual se ha formado a la mayor parte del personal de la salud. Consecuentemente este modelo educativo genera la pasividad del alumno y resalta la autoridad y el poder del profesor como poseedor del conocimiento, quien a su vez, marca el ritmo del aprendizaje del alumno; su relación con el alumno se circunscribe a sesiones discursivas o expositivas dentro de un aula donde el alumno acepta la información como verdad absoluta con una actitud subordinante y, en pocas ocasiones, se cuestiona; o bien, es ahí donde se asigna al alumno la exposición del tema, el cual, en su actitud pasiva, realiza una escasa búsqueda de información y en ocasiones se limita a expresar lo escrito en los textos sugeridos por el programa o profesor y, a su vez, éste a "corregir" o aclarar las dudas ocasionalmente generadas en el alumno. ${ }^{10}$

\section{CONCLUSIONES}

Se puede concluir que el proceso educativo en enfermería está centrado en la adquisición de habilidades técnicas y manuales para la ejecución de procedimientos y que encaja principalmente en el modelo tradicional; sus influencias se reflejan directamente en los comportamientos todavía hoy vigentes dentro de la Escuela Nacional de Enfermería y Obstetricia, a pesar de que el plan de estudios actualizado en el año 2000, implícitamente se enfoca a un modelo constructivista. este último no se lleva a cabo debido a que los estudiantes han tenido una formación tradicional, por lo que el personal de enfermería y los estudiantes son clasificadas en un nivel muy bajo de aptitud clínica, lo que sugiere una práctica profesional basada en rutinas más que en acciones reflexivas y críticas.

\section{SUGERENCIAS EN EL ÁMBITO DE ENFERMERÍA}

Se sugiere que se replanteen los planes de estudio en enfermería y que éstos promuevan una educación crítica, deliberada y participativa. Ello involucra la redefinición de roles del docente, del alumno y de la organización curricular, favoreciendo un clima organizacional creativo que incorpore asimismo una renovada y dinámica concepción de evaluación, permitiendo la formación de profesionales activos y creativos, capaces de enfrentar situaciones nuevas, de resolver problemas y de adaptarse a un mundo cambiante. Este plan tiene como expectativa, una atención en salud que refleje un conocimiento.

Para responder a las deficiencias detectadas, se plantea que la enfermería tome un papel activo al respecto, incorporando en su función educativa un diseño instruccional que conduzca a 
encontrar el camino más conveniente. El diseño de métodos y estrategias de enseñanza debe atender al desenvolvimiento de un buen pensamiento en el alumno, quien se espera sea crítico y creativo. Se debe ir motivando las formas individuales de aprender y favorecer el aprendizaje continuo para que el alumno logre ser autónomo, con una mentalidad cuestionadora y familiarizado con el proceso de aprender.

\section{BIBLIOGRAFÍA}

1. Castrillón ME. La dimensión social de la práctica de la enfermería. Medellín: Editorial Universidad de Antioquia; 1997: 19.

2. Chiondelli N, Lenise M. Educación continua en el trabajo: nuevos desafíos para los profesionales de enfermería. Rev. Investigación y Educación en enfermería [Internet]. [acceso 13 de mayo 2012]. Disponible en: http// redalyc,uamex.mx/inicvio/ArtPdfRed.jsp?jsp?;Cve=105216848011.

3. Pérez I, Castañeda C. 100 años ENEO. 1907-2006. Capítulo III. [CD interactivo]. México: ENEO-UNAM; 2006: p 5.

4. Zueras P. Historia de la Enfermería [acceso 13 de mayo 2012]. Disponible en: http://www.mixcoac.upmx.mx/document.aspx?doc=20287.
5. Chiondelli N, Lenise M. Educación continua en el trabajo: Nuevos desafíos para los profesionales de enfermería. Rev. Investigación y Educación en enfermería [acceso 17 de mayo 2012]. Disponible en: http//redalyc. uamex.mx/src/inicvio/ArtPdfRed.jsp?;Cve=105216848011

6. García E, Guillén DM, Acevedo M. La influencia del conductismo en la formación del profesional de enfermería. Razón y palabra. 2011[acceso 17 de mayo 2012]; 76. Disponible en: http://www.razonypalabra.org. mx/N/N76/varia/5a\%20entrega/46_GarciaGuillenAcevedo_V76.pdf.

7. Ávila P. Algo sobre la escuela tradicional [acceso 20 de mayo 2012]. 4-7. Disponible en: http://www.educacionemocional.cl/documentos/ educ07lacuestionescolarjpalacios.pdf

8. Rojas L. El enfoque constructivista en el currículum de enfermería: motivación, dificultades y demandas que enfrenta el o la docente. Revista Enfermería Actual en Costa Rica. 2005 [acceso 20 de mayo 2012]; 5 (9): 1-15. Disponible en: http://redalyc.uaemex.mx/pdf/448/44800901.pdf

9. Carranza AI. El constructivismo como estrategia educativa: formación profesional en enfermería. Revista de enfermería [acceso 14 de mayo 2012]. Disponible en: http://www.binasss.sa.cr/revistas/enfermeria/v30n2/art4.pdf

10. Carriles MG, Oseguera JF, Díaz Y, Gómez SA. Efecto de una estrategia educativa participativa en el desarrollo del pensamiento crítico en estudiantes de enfermería. Enfermería global Revista electrónica trimestral de enfermería. 2012; 26 [acceso 15 de Mayo 2012]. Disponible en: http:// revistas.um.es/eglobal/article/view/135891/133431. 